\title{
The metabolic syndrome: useful concept or clinical tool? Report of a WHO Expert Consultation
}

\author{
R. K. Simmons • K. G. M. M. Alberti • E. A. M. Gale $\cdot$ S. Colagiuri • J. Tuomilehto • \\ Q. Qiao • A. Ramachandran • N. Tajima • I. Brajkovich Mirchov • A. Ben-Nakhi • \\ G. Reaven • B. Hama Sambo • S. Mendis • G. Roglic
}

Received: 20 August 2009 / Accepted: 30 October 2009/Published online: 11 December 2009

(C) World Health Organization, 2009. Published by Springer-Verlag GmbH Berlin, Heidelberg, 2009. All Rights Reserved.

\begin{abstract}
This article presents the conclusions of a WHO Expert Consultation that evaluated the utility of the 'metabolic syndrome' concept in relation to four key areas: pathophysiology, epidemiology, clinical work and public
\end{abstract}

Electronic supplementary material The online version of this article (doi:10.1007/s00125-009-1620-4) contains supplementary material, which is available to authorised users.

R. K. Simmons

MRC Epidemiology Unit, Institute of Metabolic Science,

Cambridge, UK

K. G. M. M. Alberti

Endocrinology \& Metabolic Medicine, St Mary's Hospital,

London, UK

E. A. M. Gale

Diabetes and Metabolism, University Medical School Unit,

Southmead Hospital,

Bristol, UK

\section{S. Colagiuri}

Institute of Obesity, Nutrition and Exercise, University of Sydney,

Sydney, Australia

J. Tuomilehto $\cdot$ Q. Qiao

Department of Public Health, University of Helsinki,

Helsinki, Finland

J. Tuomilehto

South Ostrobothnia Central Hospital,

Seinajoki, Finland

\section{A. Ramachandran}

India Diabetes Research Foundation,

Chennai, India

\section{N. Tajima}

Department of Internal Medicine,

Jikei University School of Medicine,

Tokyo, Japan health. The metabolic syndrome is a concept that focuses attention on complex multifactorial health problems. While it may be considered useful as an educational concept, it has limited practical utility as a diagnostic or management
I. Brajkovich Mirchov

Internal Medicine Service No. 2,

Hospital Universitario de Caracas,

Caracas, Venezuela

\section{A. Ben-Nakhi}

Dasman Center for Research and Treatment of Diabetes, Kuwait City, Kuwait

G. Reaven

Division of Cardiovascular Medicine,

Stanford University School of Medicine,

Stanford, CA, USA

B. Hama Sambo

Division of Prevention and Control of Noncommunicable

Diseases, World Health Organization,

Brazzaville, Republic of the Congo

S. Mendis $\cdot$ G. Roglic $(\square)$

Department of Chronic Diseases and Health Promotion,

World Health Organization,

20 Avenue Appia,

1211 Geneva 27, Switzerland

e-mail: roglicg@who.int 
tool. Further efforts to redefine it are inappropriate in the light of current knowledge and understanding, and there is limited utility in epidemiological studies in which different definitions of the metabolic syndrome are compared. Metabolic syndrome is a pre-morbid condition rather than a clinical diagnosis, and should thus exclude individuals with established diabetes or known cardiovascular disease (CVD). Future research should focus on: (1) further elucidation of common metabolic pathways underlying the development of diabetes and CVD, including those clustering within the metabolic syndrome; (2) early-life determinants of metabolic risk; (3) developing and evaluating context-specific strategies for identifying and reducing CVD and diabetes risk, based on available resources; and (4) developing and evaluating population-based prevention strategies.

\section{Abbreviations \\ ATP III National Cholesterol Education Program Adult Treatment Panel III \\ CVD Cardiovascular disease \\ IDF International Diabetes Federation \\ NCD Non-communicable disease}

Reaven proposed a conceptual framework which linked apparently unrelated biological events into a single pathophysiological construct [3]. This hypothesis argued that insulin resistance provided a common mechanism underlying the associated abnormalities of blood pressure, HDLcholesterol, triacylglycerol and glucose tolerance. This pathophysiological concept was not intended for clinical or epidemiological use. Subsequently, a number of different definitions have been developed for this purpose by the WHO [4], the National Cholesterol Education Program Adult Treatment Panel III (ATP III) [5], the European Group for the Study of Insulin Resistance [6] and, most recently, the International Diabetes Federation (IDF) [7]. These definitions include the risk factors listed above, with the addition of (central) obesity, but rank them differently in order of importance and have sometimes used different cut-off points for the individual risk factors. More recently, the IDF and the modified ATP III definitions have become more concordant, with the remaining difference pertaining to waist measurement [8].

The rationale supporting use of the metabolic syndrome includes the following: (1) it provides a framework for research exploring a possible unifying pathophysiological basis for the observed cluster of risk factors; (2) it quantifies chronic disease risk within populations and facilitates between-country comparisons; (3) it can guide relative risk prediction and clinical management decisions; and (4) it provides an easily comprehensible public health message and reminds health professionals of the need to assess related risk factors when one risk factor is detected. Critics of the concept have pointed out that, despite an exponential increase in the number of research papers on the subject, no single unifying pathophysiological mechanism has been agreed, and the equivalence of the risk factors and their cut-off points across different populations has not been established [9]. The criteria used to diagnose the metabolic syndrome have major limitations including: the dichotomisation of risk factors; the attribution of relative as opposed to absolute risk; the differing predictive value of risk factor combinations; the inclusion of individuals with established diabetes and heart disease; and the omission of important risk factors for predicting diabetes and CVD. A formal diagnosis of the metabolic syndrome is rarely made in routine clinical practice, and the concept has not been widely adopted in national guidelines for the prediction of CVD or diabetes.

In summary, the metabolic syndrome is a widely recognised concept which focuses attention on important complex multifactorial health problems. While the concept has advantages in terms of professional and public education and awareness, critics argue that it has limitations and that its clinical utility has been over-interpreted. In light of this, a WHO Expert Consultation was undertaken in the modern concept of the metabolic syndrome began when 
November 2008 to review the concept and its utility in relation to four key areas: pathophysiology, epidemiology, clinical work and public health.

\section{Pathophysiology}

The pathogenesis of the clustering of risk factors referred to as the metabolic syndrome and its constituent elements remains unclear [10]. There is no accepted central underlying mechanism, although insulin resistance [3] and central obesity [11] have both been proposed in this role. Other central or contributory mechanisms that have been considered include: chronic activation of the immune system; disorders of the hypothalamic-pituitary-adrenal axis; altered glucocorticoid hormone action; chronic stress; and the contributions of cytokines, hormones and other molecules produced by adipocytes [10,12]. Prenatal and early-life influences might play a role [13], as may multiple gene combinations [14], possibly explaining why current definitions encompass heterogeneous phenotypes in different ethnic groups.

In the absence of a clear mechanism, interim definitions of the metabolic syndrome can only be considered provisional rather than definitive. Furthermore, given that recent research has shown a link between metabolic syndrome and other clinical conditions including liver disease [15], sleep apnoea [16] and cancer [17], it is clear that our understanding of this complex set of risk factors is limited, and that further research is needed.

\section{Epidemiology}

A considerable amount of research has been undertaken to define the epidemiology of the metabolic syndrome in different populations, and it has identified wide variations in the prevalence of metabolic syndrome between the sexes and in different countries and ethnic groups [18]. The condition has strong lifestyle determinants, which are in turn influenced by socioeconomic status, cultural habits and country-specific educational opportunities. Metabolic syndrome has become increasingly common in many populations, particularly in the developing world, and has a changing phenotype, with an increasing prevalence in younger age groups $[19,20]$. The existence of different definitions has, however, led to confusion when comparing prevalence figures and impact. Although the components of the two main versions of the metabolic syndrome are the same $[5,7]$, the specific weighting for those components that define an abnormality differs, and the manner in which they can be combined to make a positive diagnosis varies from version to version [21] (although this has recently been resolved [8]). While there is agreement on four of the central components of metabolic syndrome and conformity in the measures used to define them, there is disagreement regarding the waist circumference to be used and how this should be adjusted for use in different ethnic groups. Similarly, while prevalence estimates reached by the different definitions in a single population are often similar, there are important differences between the subpopulations which are identified as having metabolic syndrome [22, 23] and rates vary from one ethnic group to another. The implications of these findings thus remain uncertain.

While the concept of the metabolic syndrome reminds us that NCDs are multifactorial in origin, the ability of the metabolic syndrome criteria to quantify CVD and diabetes risk within a population and facilitate between-country comparisons is limited. Given the confusion surrounding different past definitions of the metabolic syndrome and the different high-risk groups identified, we suggest that it is time to call a halt to studies in which different definitions of the metabolic syndrome are compared. Consistent use of a single definition worldwide would make global comparisons of metabolic risk more meaningful. Currently, however, there is little firm basis for such a definition. We therefore suggest that research efforts focus on elucidating the mechanisms underlying the clustering of metabolic risk factors, diabetes and CVD, and developing preventive strategies, rather than on developing new or revised definitions.

\section{Clinical work}

The metabolic syndrome has been proposed as a means of identifying people at increased risk of CVD and diabetes and to guide clinical management decisions. It has been shown to predict CVD morbidity, CVD mortality, type 2 diabetes and all-cause mortality in a number of populations worldwide [12]. Risk estimates differ according to the definition used and the population subgroup to which the definition is applied. They are usually modest for the association between metabolic syndrome and CVD, and stronger for the association between metabolic syndrome and type 2 diabetes [12, 24]. Metabolic syndrome criteria are, however, outperformed by traditional cardiovascular risk prediction algorithms, such as Framingham [25], and do not enhance risk prediction [22, 24]. This is unsurprising as the metabolic syndrome can only indicate relative risk. Similarly, the metabolic syndrome confers an elevated risk of incident diabetes, but not more than some of its individual components: e.g. fasting glucose conveys a greater risk of incident diabetes than the metabolic syndrome [26]. This has led critics to argue that the constellation of risk factors for the metabolic syndrome does not offer more than the sum of its parts in terms of 
diagnosis and management [9]. Furthermore, current definitions are not very discriminating, and from $10 \%$ to almost half of the world's adult population will be identified as having the metabolic syndrome depending on which definition is used [22]. Finally, the clinical utility of the metabolic syndrome is confused by the existence of different definitions which identify different risk populations [22].

The construct of the metabolic syndrome diagnostic criteria has inherent limitations which impact on its clinical usefulness. Six are described below.

Dichotomisation of the diagnosis of metabolic syndrome and of risk factors used to define metabolic syndrome Dichotomisation is a common feature of diagnostic criteria for many diseases, including diabetes, and was employed in the metabolic syndrome for ease of use in primary care. However, the use of discrete thresholds to define abnormalities is artificial and discards crucial information about the magnitude of the risk factors [22]. The association of risk factors such as blood pressure and cholesterol with CVD is continuous and does not show any point at which risk clearly increases. Furthermore, the summation of components into a unitary diagnosis assumes that each dichotomised risk factor carries the same risk, yet some factors included in each definition are more strongly predictive of CVD than others.

Omission of established risk factors The metabolic syndrome does not include other important risk factors for predicting diabetes or CVD, such as age, sex, family history, socioeconomic status, ethnicity, current treatment, previous CVD events and LDL-cholesterol, or important behavioural variables such as smoking and physical activity.

Metabolic syndrome describes relative risk as opposed to absolute risk An individual's risk of developing diabetes or CVD is related to baseline risk. For example, a fourfold increase in relative risk in an individual with low baseline risk is likely to be less relevant than a twofold increase in absolute risk in an individual with high baseline risk. As such, absolute risk is a more useful measure in clinical practice. It should be noted that metabolic syndrome was not intended for use as an absolute risk predictor but rather as an indicator of relative risk.

Heterogeneity among individuals diagnosed with the metabolic syndrome There are 16 different ways to diagnose the metabolic syndrome using the ATP III definition and eleven different ways to diagnose the metabolic syndrome using the IDF definition. As such, there is interindividual heterogeneity of the risk-factor components on which a diagnosis of metabolic syndrome is made, and each combination identifies a different risk population [23].

Cardiovascular risk varies according to the risk factor combination used to diagnose metabolic syndrome in an individual The risk of CVD and diabetes is not equivalent across the different risk factor combinations which constitute a metabolic syndrome diagnosis [27]. This phenomenon is linked to the fact that although we often observe a clustering of risk factors, each component has multiple causes, some of which do not involve the metabolic syndrome but still increase risk for diabetes and CVD.

Defining obesity within metabolic syndrome criteria The IDF definition uses waist circumference as a useful criterion and entry point for further risk assessment, though there have been difficulties establishing ethnic-specific cutoff points. However, measurement of BMI and waist circumference often provide comparable estimates of the relationship between degree of adiposity and CVD risk [28], and each may make independent contributions in this regard [29]. Consequently, either measurement can provide a simple approach to identifying individuals at risk for developing the component parts of the metabolic syndrome that contribute to CVD risk.

The metabolic syndrome has not generally been adopted by national guidelines for the prediction of CVD or diabetes. Furthermore, a formal diagnosis of metabolic syndrome using any of the current definitions is rarely made in routine clinical practice, and the syndrome does not demand a specific treatment. There are currently no therapies - other than lifestyle modification-that specifically address the cluster of metabolic syndrome risk factors, and each individual risk factor requires separate treatment. Consequently, we emphasise that metabolic syndrome is a pre-morbid condition rather than a clinical diagnosis, and should thus exclude individuals with established diabetes or known cardiovascular disease. Although metabolic syndrome can predict diabetes and CVD, the construct was never intended for use as a detailed risk predictor, and there are other tools available which provide a measure of absolute risk within defined populations. In resource-poor settings, the use of WHO risk charts could be useful to inform risk stratification [30]. More developed health settings can adopt a pragmatic stepwise approach to risk stratification, where routine data or simple questionnaires are used to identify those at increased risk (without the need for laboratory measures). Individuals identified could then be invited for a short health assessment, including blood sampling, where absolute CVD risk can be quantified and appropriate preventive strategies recommended. As health information systems evolve, more sophisticated tools could be developed for risk stratification. 


\section{Public health}

While there is very little research directly addressing the impact of metabolic syndrome on public health and/or health promotion activities, the introduction of the concept and the consequent surge of interest in the syndrome have stimulated valuable thinking and research across different disciplines [22]. Anecdotal evidence suggests that the metabolic syndrome concept has: (1) provided an easily comprehensible public health message; (2) educated health professionals on the importance of risk factor clustering and the need to assess related risk factors when one risk factor is detected; and (3) encouraged health professionals not to focus simply on diabetes or CVD. In Japan, for example, the Government recently introduced a national screening programme using the metabolic syndrome concept as a single point of entry to identify people at high risk who might benefit from an intervention to reduce CVD risk [31]. However, while many patients and practitioners still need educating on the multifactorial nature of NCDs, the same prevention and management strategies are recommended for both metabolic syndrome and its constituent parts (e.g. a healthy diet, regular physical activity, smoking cessation and weight loss/control, plus pharmacological intervention where necessary). Consequently, unless currently used in national or local public health campaigns, we do not encourage further introduction of the metabolic syndrome concept.

Effective lifestyle and pharmaceutical approaches to the prevention of diabetes and CVD in high-risk individuals are well established [32-35]. Each country should aim to develop context-specific policies for the identification and reduction of diabetes and CVD risk, based on the resources available. Although the cost-effectiveness of interventions to prevent and control non-communicable diseases remains unclear, a full range of options should be considered, including lifestyle modification and highquality low-cost generic medications. While the metabolic syndrome and related risk stratification approaches focus attention on individuals at high risk of future disease, the importance of population-based prevention strategies should not be overlooked.

\section{Conclusions}

The metabolic syndrome is a concept that focuses attention on complex multifactorial health problems. While it may be considered useful as an educational concept, it has limited practical utility as a diagnostic or management tool. Further efforts to redefine it are inappropriate in the light of current knowledge and understanding, and epidemiological studies in which different definitions of the metabolic syndrome are compared are of limited utility.

The metabolic syndrome should be considered a pre-morbid condition and should thus exclude individuals with established diabetes or known cardiovascular disease.

Metabolic syndrome should not be applied as a clinical diagnosis.

Each country should aim to develop cost-effective context-specific identification and reduction strategies for diabetes and CVD risk, based on available resources. These should be complemented with population-based prevention strategies focusing on the control and reduction of behavioural and metabolic risk factors by targeting their key determinants.

Future research should focus on: (1) further elucidation of common metabolic pathways underlying the development of diabetes and CVD, including those clustering within the metabolic syndrome; (2) early-life determinants of metabolic risk; (3) developing and evaluating contextspecific identification and reduction strategies for diabetes and CVD risk, based on available resources; and (4) developing and evaluating population-based prevention strategies.

\section{The consultation}

The experts for the consultation were invited on the basis of their published work on the metabolic syndrome, with an attempt to ensure participation from all six WHO regions, to balance the sex distribution and represent different approaches of the authoritative national and international bodies. The conclusions were unanimously accepted by the participants. The list of experts invited to participate in the consultation is presented in Electronic supplementary material (ESM) Table 1.

Acknowledgements The contribution of the experts who participated in the consultation is greatly appreciated. We are grateful for the background papers prepared by S. Colagiuri, E. A. M. Gale, J. Shaw and R. K. Simmons. We thank K. G. M. M. Alberti and E. A. M. Gale for co-chairing the consultation, and R. K. Simmons for acting as rapporteur. WHO thanks the Japanese Government for financing the consultation.

Duality of interest K. G. M. M. Alberti has received fees for consultancy from AstraZeneca, Boehringer Ingelheim, Merck and Servier. J. Tuomilehto has received research funding and/or consultancy fees from AstraZeneca, Bayer Pharma and Merck. S. Colagiuri has received consultancy fees and grants from Merck Sharp \& Dohme, GlaxoSmithKline, Lilly, sanofi-aventis and Novo Nordisk. N. Tajima has received lecture fees from the pharmaceutical industry. The other authors declare that there is no duality of interest associated with this manuscript. The views expressed within this paper are those of the authors and not necessarily those of WHO. 


\section{References}

1. World Health Organization (2008) 2008-2013 Action plan for the global strategy for the prevention and control of noncommunicable diseases. WHO, Geneva

2. United Nations (2008) The Millennium Development Goals report 2008. United Nations, New York

3. Reaven GM (1988) Banting Lecture 1988. Role of insulin resistance in human disease. Diabetes 37:1595-1607

4. World Health Organization (1999) Definition, diagnosis and classification of diabetes mellitus and its complications. Part 1: diagnosis and classification of diabetes mellitus. WHO, Geneva

5. Grundy SM, Brewer HB Jr, Cleeman JI, Smith SC Jr, Lenfant C (2004) Definition of metabolic syndrome: Report of the National Heart, Lung, and Blood Institute/American Heart Association conference on scientific issues related to definition. Circulation 109:433-438

6. Balkau B, Charles MA (1999) Comment on the provisional report from the WHO consultation. European Group for the Study of Insulin Resistance (EGIR). Diabet Med 16:442-443

7. Alberti KG, Zimmet P, Shaw J (2005) The metabolic syndromea new worldwide definition. Lancet 366:1059-1062

8. Alberti KG, Eckel RH, Grundy SM et al (2009) Harmonizing the Metabolic Syndrome. A Joint Interim Statement of the International Diabetes Federation Task Force on Epidemiology and Prevention; National Heart, Lung, and Blood Institute; American Heart Association; World Heart Federation; International Atherosclerosis Society; and International Association for the Study of Obesity. Circulation 120:1640-1645

9. Kahn R, Buse J, Ferrannini E, Stern M (2005) The metabolic syndrome: time for a critical appraisal. Joint statement from the American Diabetes Association and the European Association for the Study of Diabetes. Diabetologia 48:1684-1699

10. Eckel RH, Grundy SM, Zimmet PZ (2005) The metabolic syndrome. Lancet 365:1415-1428

11. Carr DB, Utzschneider KM, Hull RL et al (2004) Intra-abdominal fat is a major determinant of the National Cholesterol Education Program Adult Treatment Panel III criteria for the metabolic syndrome. Diabetes 53:2087-2094

12. Ford ES (2005) Risks for all-cause mortality, cardiovascular disease, and diabetes associated with the metabolic syndrome: a summary of the evidence. Diabetes Care 28:1769-1778

13. Fernandez-Twinn DS, Ozanne SE (2006) Mechanisms by which poor early growth programs type- 2 diabetes, obesity and the metabolic syndrome. Physiol Behav 88:234-243

14. Sjogren M, Lyssenko V, Jonsson A et al (2008) The search for putative unifying genetic factors for components of the metabolic syndrome. Diabetologia 51:2242-2251

15. Kotronen A, Yki-Jarvinen H (2008) Fatty liver: a novel component of the metabolic syndrome. Arterioscler Thromb Vasc Biol 28:27-38

16. Vgontzas AN, Bixler EO, Chrousos GP (2005) Sleep apnea is a manifestation of the metabolic syndrome. Sleep Med Rev 9:211-224

17. Russo A, Autelitano M, Bisanti L (2008) Metabolic syndrome and cancer risk. Eur J Cancer 44:293-297

18. Wild SH, Byrne CD (2005) The global burden of the metabolic syndrome and its consequences for diabetes and cardiovascular disease. In: Wild SH, Byrne CD (eds) The metabolic syndrome. Wiley, Chichester, pp 1-43

19. Weiss R, Dziura J, Burgert TS et al (2004) Obesity and the metabolic syndrome in children and adolescents. N Engl J Med 350:2362-2374

20. Esmaillzadeh A, Mirmiran P, Azadbakht L, Etemadi A, Azizi F (2006) High prevalence of the metabolic syndrome in Iranian adolescents. Obesity 14:377-382

21. Reaven GM (2006) The metabolic syndrome: is this diagnosis necessary? Am J Clin Nutr 83:1237-1247

22. Eddy DM, Schlessinger L, Heikes K (2008) The metabolic syndrome and cardiovascular risk: implications for clinical practice. Int J Obes 32(Suppl 2):S5-10

23. Lee CM, Huxley RR, Woodward M et al (2008) The metabolic syndrome identifies a heterogeneous group of metabolic component combinations in the Asia-Pacific region. Diabetes Res Clin Pract 8:377-380

24. Sattar N, McConnachie A, Shaper AG et al (2008) Can metabolic syndrome usefully predict cardiovascular disease and diabetes? Outcome data from two prospective studies. Lancet 371:19271935

25. Kohli P, Greenland P (2006) Role of the metabolic syndrome in risk assessment for coronary heart disease. JAMA 295:819-821

26. Cameron AJ, Magliano DJ, Zimmet PZ et al (2008) The metabolic syndrome as a tool for predicting future diabetes: the AusDiab study. J Intern Med 264:177-186

27. Mancia G, Bombelli M, Corrao G et al (2007) Metabolic syndrome in the Pressioni Arteriose Monitorate E Loro Associazioni (PAMELA) study: daily life blood pressure, cardiac damage, and prognosis. Hypertension 49:40-47

28. Stevens J, McClain JE, Truesdale KP (2008) Selection of measures in epidemiologic studies of the consequences of obesity. Int J Obes 32(Suppl 3):S60-S66

29. Canoy D (2008) Distribution of body fat and risk of coronary heart disease in men and women. Curr Opin Cardiol 23:591-598

30. Mendis S, Lindholm LH, Mancia G et al (2007) World Health Organization (WHO) and International Society of Hypertension (ISH) risk prediction charts: assessment of cardiovascular risk for prevention and control of cardiovascular disease in low and middle-income countries. J Hypertens 25:1578-1582

31. Kohro T, Furui Y, Mitsutake N et al (2008) The Japanese national health screening and intervention program aimed at preventing worsening of the metabolic syndrome. Int Heart J 49:193-203

32. Ramachandran A, Snehalatha C, Mary S, Mukesh B, Bhaskar AD, Vijay V (2006) The Indian Diabetes Prevention Programme shows that lifestyle modification and metformin prevent type 2 diabetes in Asian Indian subjects with impaired glucose tolerance (IDPP-1). Diabetologia 49:289-297

33. Tuomilehto J, Lindstrom J, Eriksson JG et al (2001) Prevention of type 2 diabetes mellitus by changes in lifestyle among subjects with impaired glucose tolerance. N Engl J Med 344:1343-1350

34. Knowler WC, Barrett-Connor E, Fowler SE et al (2002) Reduction in the incidence of type 2 diabetes with lifestyle intervention or metformin. N Engl J Med 346:393-403

35. Eyre H, Kahn R, Robertson RM et al (2004) Preventing cancer, cardiovascular disease, and diabetes: a common agenda for the American Cancer Society, the American Diabetes Association, and the American Heart Association. Circulation 109:3244-3255 